\title{
Parâmetros genéticos para características de carcaça avaliadas por ultrassonografia em bovinos da raça Guzerá
}

\section{RESUMO}

A partir das observações de 1.325 animais ( $90,4 \%$ de machos e $9,6 \%$ de fêmeas) e do pedigree de 6.642 animais da raça Guzerá foram estimados os parâmetros genéticos para o peso corporal e as características área de olho de lombo e espessura de gordura na costela e na garupa, avaliadas por meio da técnica de ultrassonografia. Os componentes de (co)variância foram estimados pelo método da máxima verossimilhança restrita, utilizando-se o aplicativo MTDFREML. Foram utilizados, para as estimativas de repetibilidade e herdabilidade, modelos unicaracterística e, para as correlações genéticas e fenotípicas entre as características, modelos bicaracterísticas. As estimativas de repetibilidade (erros-padrão) foram $0,44(0,10)$ para peso corporal, $0,39(0,10)$ para área de olho de lombo, $0,75(0,06)$ para espessura de gordura na costela e $0,49(0,08)$ para espessura de gordura na garupa. As estimativas de herdabilidade, respectivamente a partir de modelos uni e bicaracterísticas, foram $0,42(0,11)$ e $0,41(0,11)$ para peso corporal, $0,35(0,09)$ e $0,34(0,09)$ para área de olho de lombo, $0,20(0,08)$ e $0,32(0,02)$ para espessura de gordura na garupa e $0,05(0,06)$ e $0,10(0,08)$ para espessura de gordura na costela. As estimativas de correlações genéticas foram $0,79(0,09)$ entre o peso corporal e a área de olho de lombo; $0,20(0,08)$ entre o peso corporal e a espessura de gordura na garupa; $0,05(0,06)$ entre a área de olho de lombo e a espessura de gordura na costela; $0,02(0,27)$ entre a área de olho de lombo e a espessura de gordura na garupa; e $0,64(0,22)$ entre as duas medidas de espessura de gordura. Os resultados indicam que é uma mensuração suficiente para a adequada avaliação das características área de olho de lombo e espessura de gordura na carcaça e que a seleção direta para essas características pode resultar em carcaças mais musculosas e de melhor acabamento. Indica, ainda, ausência de antagonismo genético entre a seleção para peso corporal e características área de olho de lombo e espessura de gordura na carcaça.

Palavras-chave: bovino de corte, parâmetros genéticos, peso corporal, características de carcaça

\begin{abstract}
Genetic parameters were estimated for body weight and real-time ultra-sound loin-eye area, rump fat thickness, and back fat thickness using data from 1,325 yearling Guzera cattle (90.4\% bulls and 9.6\% heifers) and pedigree structure with 6,642 animals. Variance and covariance components were estimated using REML methodology and MTDFREML software. Single trait animal models were used to estimate repeatability and heritability for the four traits. Multiple traits animal models were used to estimate genetic correlations among the traits. Repeatability estimates (standard errors) were 0.44(0.10) for body
\end{abstract}

Recebido em 13 de agosto de 2008

Aceito em 20 de janeiro de 2009

E-mail: helioufla@click21.com.br 
weight, 0.39(0.10) for loin-eye area, 0.75(0.06) for rump fat thickness, and 0.49(0.08) for back fat thickness. Heritability estimates were 0.42(0.11) and 0.41(0.11) for body weight, 0.35(0.09) and $0.34(0.09)$ for loin-eye area, 0.20(0.08) and 0.32(0.08) for back fat thickness, and 0.05(0.06) and $0.10(0.08)$ for rump fat thickness, respectively from single and multiple traits models. Genetic correlation estimates were 0.79(0.09) between body weight and loin-eye area, 0.20(0.08) between body weight and back fat thickness, 0.05(0.06) between loin-eye area and rump fat thickness, 0.02(0.27) between loin-eye area and back fat thickness and 0.64(0.22) between the two measurements of fat thickness. In order to evaluate carcass traits, results suggested that repeated real-time ultra-sound measurements are not needed and that direct selection for these traits might be effective. In addition, there is no genetic antagonism between selection for body weight and carcass traits.

Keywords: beef cattle, genetic parameters, body weight, carcass traits

\section{INTRODUÇÃO}

Tradicionalmente, os programas de melhoramento genético de bovinos de corte no Brasil têm considerado características de desenvolvimento e de reprodução (Marcondes et al., 2000; Simonelli et al., 2004). Mais recentemente, alguns programas de melhoramento genético da raça Nelore têm incluído a predição do mérito genético para características de carcaça, avaliadas por ultrassonografia, e publicado diferenças esperadas na progênie (DEPs) de touros e matrizes para essas características (Lobo et al., 2006).

Segundo Araújo (2003), a avaliação genética de características associadas ao rendimento e à qualidade da carcaça e a seleção para essas características são importantes para melhorar a carcaça do zebu brasileiro. Para Bailey et al. (1986) e Bertrand et al. (2001), a espessura de gordura subcutânea e a área do músculo Longissimus dorsi ou área de olho de lombo, mensuradas por ultrassonografia, são características de eleição. Ainda, segundo Araújo (2003), as estimativas de parâmetros genéticos para essas características de animais da raça Nelore indicam a possibilidade de resposta direta à seleção e têm propiciado a estimação de DEPs e seu emprego na escolha de animais para a reprodução (Lobo et al., 2006). Entretanto, para a raça Guzerá, não foram encontrados na presente revisão estudos que avaliem os aspectos genéticos das características de carcaça obtidas por meio da técnica de ultrassonografia.

O objetivo do presente trabalho foi a obtenção de estimativas de repetibilidade e herdabilidade para as características de carcaça, área de olho de lombo e espessura de gordura na garupa e na costela, e a de correlações genéticas e fenotípicas entre essas característica e o peso corporal de animais da raça Guzerá.

\section{MATERIAL E MÉTODOS}

Foram utilizados 1.325 animais da raça Guzerá (90,4\% machos e 9,6\% fêmeas), nascidos em quatro fazendas do estado de São Paulo, no período de setembro de 2002 a junho de 2005 . Os animais foram pesados e avaliados, por ultrassonografia, para as características da carcaça quando tinham idade média de 17,6 meses, variando entre 10 e 32 meses. Nesta amplitude de idade, $40,2 \%$ dos animais foram medidos uma única vez, $49,8 \%$ tiveram duas medidas e $10,0 \%$ tiveram três ou quatro medidas.

As fazendas de origem dos animais, com sua contribuição para o conjunto de dados e localização, foram: (1) fazenda Perfeita União (21\% dos animais) e (2) fazenda Alvorada (30\% dos animais), localizadas no município de Pirajuí; (3) fazenda Negrinha (9\% dos animais), localizada no município de Parapuã; e (4) fazenda São Judas Tadeu $(40 \%$ do total de animais), localizada no município de Porto Feliz. As regiões das fazendas são predominantemente tropicais úmidas com prevalência de plantas forrageiras dos gêneros Brachiaria e Panicum, e precipitação anual média variando de 1.300 a $1.600 \mathrm{~mm}$. Todos os animais tiveram livre acesso a água e suplementação mineral durante todo o período experimental. O manejo nutricional dos animais foi classificado como: (1) exclusivamente a pasto; (2) pastejo, com suplementação volumosa; e (3) confinamento, com dietas de alta energia.

De acordo com a fazenda, os dados foram coletados em oito datas, no período de 2004 a 
2006, assim definidas: (1) mês de junho de 2004, nas fazendas 1 e 4; (2) mês de setembro de 2004, nas fazendas 1, 3 e 4; (3) mês de dezembro de 2004, na fazenda 1 ; (4) meses de março e maio de 2005, nas fazendas 1 e 2; (5) meses de julho e setembro de 2005, nas fazendas 1,2 e 4; (6) mês de maio de 2005, na fazenda 4; (7) mês de março de 2006, nas fazendas 1 e 2; e (8) mês de junho de 2006, nas fazendas 2 e 4.

As características de carcaça consideradas foram: área de olho de lombo (AOL), avaliada a partir do perímetro do músculo Longissimus dorsi obtido da imagem digitalizada, com o transdutor posicionado transversalmente ao animal no espaço entre a $12^{\mathrm{a}}$ e a $13^{\mathrm{a}}$ costelas; espessura de gordura na costela (EG), obtida com o transdutor posicionado paralelamente à espinha dorsal do animal, entre a $12^{\mathrm{a}}$ e $13^{\mathrm{a}}$ costelas; e espessura de gordura na garupa (EGP8), obtida com o transdutor posicionado na junção dos músculos Biceps femoris e Gluteus medium, entre o ísquio e o íleo. Para as leituras ultrassonográficas, feitas após a pesagem, os animais foram contidos em brete restrito e em área fechada, na tentativa de oferecer relativo conforto e relaxamentos para melhor qualidade das imagens. Todas as medidas de ultrassonografia foram feitas por um mesmo técnico, credenciado da Aval Serviços Tecnológicos S.A. O aparelho utilizado foi o Aloka $500 \mathrm{~V}^{1}$, com um transdutor linear de 3,5 $\mathrm{MHz}$ com $17,2 \mathrm{~cm}$.

As imagens foram digitalizadas e armazenadas por meio de um sistema de captura de imagem ${ }^{2}$. A digitalização foi feita por meio do programa Image- $\mathrm{J}^{3}$. As coletas no campo e a interpretação das imagens foram efetuadas por técnicos experientes e membros da equipe da Aval Serviços Tecnológicos S.A.

Para a consistência dos dados e a formação dos grupos contemporâneos (GC), foi utilizado o aplicativo SAS/1998. De acordo com as classes de sexo, ano e fazenda de nascimento, manejo alimentar e data de medição, foram identificados 43GC, com, no mínimo, três animais.

Os componentes de (co)variância, as repetibilidades, as herdabilidades $\mathrm{e}$ as

\footnotetext{
${ }^{1}$ Aloka Co. Ltd. - Wallingford, EUA

${ }^{2}$ Blackbox, Biotronics - Ames, EUA.

${ }^{3}$ National Institutes of Health, EUA.
}

correlações genéticas foram estimados para as características peso corporal, AOL, EG e EGP8, considerando os efeitos fixos de GC e da covariável idade do animal (linear e quadrática) em modelo animal, uni e bicaracterísticas, utilizando o método da máxima verossimilhança restrita livre de derivadas, com o aplicativo MTDFREML (Boldman et al., 1995). Nestas análises foi considerada a matriz de parentesco até os animais-base (pais e mães desconhecidos), contendo 6.642 animais. Em cada ciclo de iteração, a variância dos valores do logaritmo da função de verossimilhança no simplex foi calculada. O critério de convergência adotado foi igual ou superior a $10^{-9}$. Várias reinicializações foram executadas no sentido de se assegurar a convergência no máximo global da função de verossimilhança.

Os valores iniciais para as análises unicaracterística foram obtidos da literatura. Para as análises bicaracterísticas foram utilizados como valores iniciais os parâmetros obtidos nas análises unicaracterística (variâncias) e valores da literatura para as covariâncias.

Para obtenção das estimativas de herdabilidade e de repetibilidade, o modelo unicaracterística utilizado, na forma matricial, foi o seguinte:

$y=X \beta+Z a+S p_{e}+e$, em que:

$y=$ vetor das variáveis dependentes;

$\beta=$ vetor para os efeitos fixos (GC e idade linear e quadrática);

$a=$ vetor de efeitos genéticos dos animais;

$p_{e}=$ vetor de efeitos permanentes dos animais;

$e=$ vetor de erros aleatórios residuais associados às observações; e

$X, Z$ e $S$ são matrizes de incidência para cada efeito.

Para obtenção das estimativas de correlações genéticas entre as características, foi considerada apenas uma observação para cada animal, escolhendo-se, no caso de animais com mais do que uma observação, aquela em que a idade do animal estava mais próxima da média (17,6 meses). O modelo genético bicaracterísticas adotado foi: 
$\left[\begin{array}{l}\mathrm{y}_{1} \\ \mathrm{y}_{2}\end{array}\right]=\left[\begin{array}{cc}\mathrm{X}_{1} & 0 \\ 0 & \mathrm{X}_{2}\end{array}\right] \cdot\left[\begin{array}{l}\beta_{1} \\ \beta_{2}\end{array}\right]+\left[\begin{array}{ll}\mathrm{Z}_{1} & 0 \\ 0 & \mathrm{Z}_{2}\end{array}\right] \cdot\left[\begin{array}{l}\mathrm{a}_{1} \\ \mathrm{a}_{2}\end{array}\right]+\left[\begin{array}{l}\mathrm{e}_{1} \\ \mathrm{e}_{2}\end{array}\right]$,

em que:

$y_{1}=$ vetor da variável dependente 1

$y_{2}=$ vetor da variável dependente 2 ;

$\beta_{1}=$ vetor para os efeitos fixos associados à característica 1 ;

$\beta_{2}=$ vetor para os efeitos fixos associados à característica 2 ;

$a_{1}=$ vetor de efeitos genéticos dos animais para a característica 1 ;

$a_{2}=$ vetor de efeitos genéticos dos animais para a característica 2;

$e_{1}=$ vetor de erros aleatórios residuais associados às observações da característica 1 ;

$e_{2}=$ vetor de erros aleatórios residuais associados às observações da característica 2 ;

vetor de efeitos genéticos dos animais para a característica 2; e

$X_{1}, X_{1}, Z_{1}$ e $Z_{2}$ são matrizes de incidência para cada efeito e característica.

Para os dois modelos, foram assumidos $\mathrm{E}(y)=X \beta$; $\mathrm{E}(a)=0, \quad \mathrm{E}\left(p_{e}\right)=0, \quad \mathrm{E}(e)=0 ; \quad \operatorname{Cov}\left(a, p_{e}\right)=0, \quad$ Var $(a)={ }_{\mathrm{A}_{\sigma_{\mathrm{a}}^{2}}^{2}}$, em que A é a matriz de parentesco e $\sigma_{a}^{2}$, a variância genética aditiva.

\section{RESULTADOS E DISCUSSÃO}

A amplitude na variação da idade dos animais por ocasião das mensurações foi 22 meses (de 10 a 32 meses, com $72 \%$ dos animais com idade entre 14 e 20 meses). Apesar de alguns animais estarem muito pesados (Tab. 1), a maioria (75\%) estava sob regime de pastejo, com suplementação volumosa e foram avaliados por volta de $405 \mathrm{~kg}$ de peso vivo, aos 18 meses de idade. Além da idade, análises subsequentes evidenciaram que outros fatores de meio contribuíram para a variação observada para as características estudadas. A amplitude na espessura de gordura na costela e na garupa evidencia o potencial da raça Guzerá para acabamento de carcaça. Saliente-se que usualmente os animais zebuínos criados no pasto não têm a possibilidade de expressar seu máximo potencial genético para acúmulo de gordura subcutâneo devido à limitada condição nutricional das pastagens de clima tropical (Araújo, 2003).

A visualização do efeito conjunto da idade e do manejo alimentar sobre o peso corporal e sobre as três características da carcaça é apresentada na Fig. 1. Foi observado que a deposição de gordura na garupa ocorreu de forma linear e mais acentuada do que na costela. Para cada mês de idade houve, em média, aumento de $0,14 \mathrm{~mm}$ de gordura na garupa. Segundo Luchiari Filho (2000), a gordura é o tecido mais variável da carcaça, tanto em quantidade quanto em distribuição. De acordo com Berg e Butterfield (1986), a deposição de gordura ocorre primeiramente no traseiro e dianteiro, avançando em direção à coluna vertebral. Ainda, à exceção da espessura de gordura na garupa, neste estudo os máximos para as demais caractertísticas ocorreram no limite superior da idade (32 meses) ou fora dele, o que evidencia que estes animais ainda estão em crescimento.

Tabela 1. Número de observações $(\mathrm{N})$, média aritmética $(\mu)$, desvio-padrão (DP), coeficiente de variação (CV) e valores mínimo (Mín.) e máximo (Máx.) do peso corporal e das características de carcaça avaliadas por ultrassonografia de animais da raça Guzerá

\begin{tabular}{lcccccc}
\hline \multicolumn{1}{c}{ Característica } & $\mathrm{N}$ & $\mu$ & $\mathrm{DP}$ & $\mathrm{CV}(\%)$ & Mín & Máx \\
\hline Peso $(\mathrm{kg})$ & 1.325 & 391,0 & 78,1 & 58,4 & 210,2 & 684,2 \\
Área de olho de lombo $\left(\mathrm{cm}^{2}\right)$ & 1.319 & 58,10 & 11,62 & 19,2 & 26,48 & 97,62 \\
Espessura de gordura na costela $(\mathrm{mm})$ & 1.319 & 2,60 & 1,74 & 46,1 & 0,87 & 18,80 \\
Espessura de gordura na garupa $(\mathrm{mm})$ & 1.321 & 3,60 & 2,29 & 42,2 & 0,87 & 21,82 \\
\hline
\end{tabular}

Os resultados relacionados à importância dos efeitos não genéticos sobre as características da carcaça estão em sintonia com os encontrados por Yokoo (2005). Para o autor, na predição das DEPs para as características da carcaça, é de fundamental importância ter sob controle os grupos contemporâneos e de manejo, assim como a padronização da idade da medida de ultrassonografia

As estimativas de repetibilidades (erros-padrão) foram $0,44(0,10)$ para $o$ peso corporal; 
$0,39(0,10)$ para a AOL, $0,75(0,06)$ para a EG e $0,49(0,08)$ para a EGP8. Os valores encontrados para peso corporal, área de olho de lombo e espessura de gordura na garupa foram moderados e estão de acordo com a literatura. O valor estimado para a espessura de gordura na costela foi maior do que o observado para a espessura de gordura na garupa e está entre os maiores encontrados na literatura para animais Bos indicus (Figueiredo, 2001; Yokoo, 2005). Devese salientar que os valores de repetibilidade obtidos neste estudo representam estimativas de parâmetros das características, obtidos de dados de ultrassonografia mensurados em um mesmo animal com intervalos médios de três meses. Esta situação difere da de outros estudos que utilizaram intervalos de dias ou horas, com o intuito de aferir a precisão da mensuração. Portanto, se as estimativas de repetibilidade desse experimento tivessem sido obtidas a partir de medidas em intervalos de tempo mais curtos, poderiam ter sido mais expressivas. Os resultados aqui observados indicaram ser pequeno o ganho com a adoção de mensurações

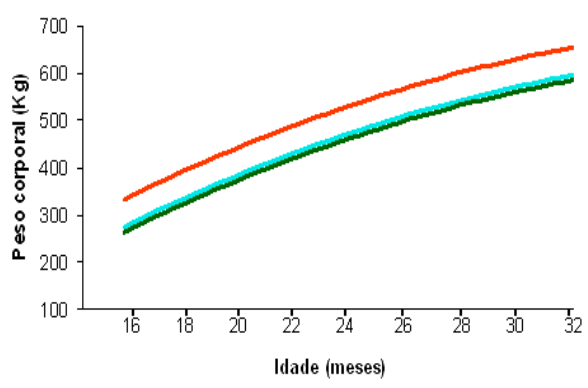

-Manejo $1 ; y=31,0980669+27,3286572 x-0,3027174 x^{2}$ -Manejo $2 ; y=21,1305230+27,3286572 x-0,3027174 x^{2}$ -Manejo $3 ; y=90,5461626+27,3286572 x-0,3027174 x^{2}$

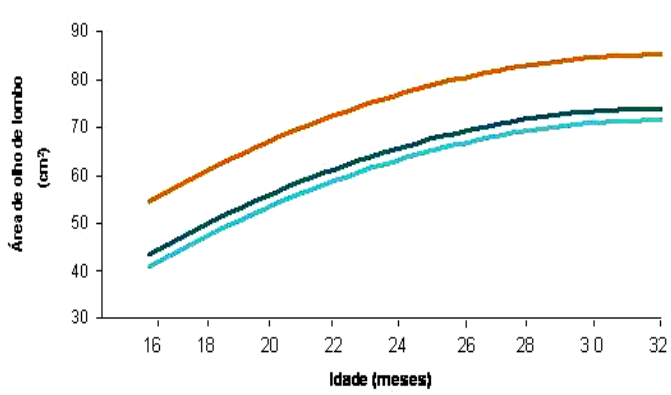

- Manejo $1 ; y=5,36944101+4,01118384 x-0,06078041 x_{2}^{2}$ -Manejo $2 ; y=7,83644702+4,01118384 x-0,06078041 x_{2}^{2}$ - Manejo $3 ; y=19,0192037+4,01118384 x-0,06078041 x^{2}$ múltiplas quando for feita a seleção e estimados valores genéticos para características da carcaça.

As estimativas de herdabilidade (erros-padrão) obtidas com análises unicaracterísticas foram $0,42(0,11)$ para o peso corporal; $0,35(0,09)$ para a AOL, $0,20(0,08)$ para a EG e $0,05(0,06)$ para a EGP8. As estimativas de herdabilidade e de correlações genéticas e fenotípicas, a partir de análises bicaracterísticas são apresentadas na Tab. 2.

Com exceção da herdabilidade estimada para a espessura de gordura na costela, os resultados encontrados estão dentro da amplitude de estimativas obtidas por Moser et al. (1998), Ritchie (2001) e Reverter et al. (2003) que estimaram as herdabilidades e correlações genéticas para as características de carcaça para várias raças Bos taurus. Nos diversos estudos, as estimativas foram consideradas de média-alta, variando de 0,29 a 0,64, dependendo da característica avaliada, do sexo e da raça.

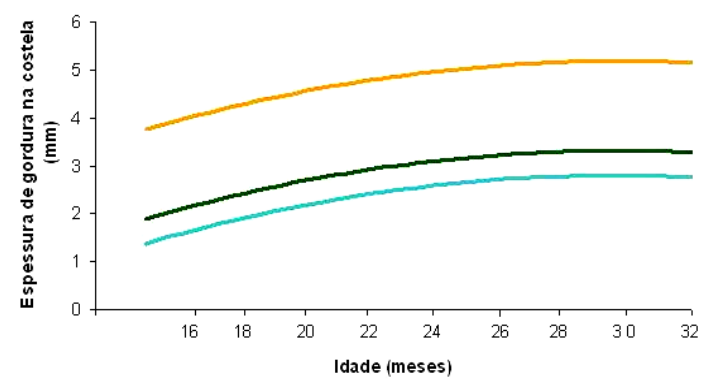

-Manejo 1; $y=1,851199783+0,229140512 x-0,003954361 x^{2}$ —Manejo 2; $y=-0,0118603+0,229140512 x-0,003954361 x^{2}$ - Manejo $3 . y=0,5225996+0,229140512 x-0,003954361 x^{2}$

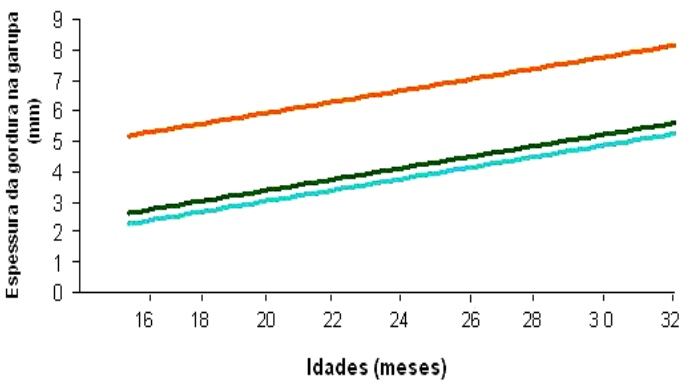

Manejo 1; $y=0,92139540+0,1351510370 x$
Manejo 2; $y=1,27713870+0,1351510370 x$
Manejo 3; $y=3,81739179+0,1351510370 x$

Figura 1. Representação gráfica do efeito de idade do animal e do manejo alimentar (1: exclusivamente a pasto; 2 : pastejo, com suplementação volumosa; e 3: confinamento, com dieta de alta energia) sobre o peso corporal, a área de olho de lombo e as espessuras de gordura na costela e na garupa de animais da raça Guzerá. 
Tabela 2. Estimativas das herdabilidades (diagonal, em negrito), dos respectivos erros-padrão (EP) e das correlações genéticas (acima da diagonal) e fenotípicas (abaixo da diagonal) para as características peso corporal e de carcaça em animais da raça Guzerá obtidas por análise bicaracterística

\begin{tabular}{lcccccc}
\multicolumn{1}{c}{ Característica } & Peso & Área de & & \multicolumn{2}{c}{ Espessura de gordura } \\
\cline { 2 - 3 } & corporal & olho de lombo & Garupa & Costela \\
\hline Peso corporal & $0,41(0,11)$ & $0,79(0,09)$ & & $0,20(0,08)$ & NE \\
Área de olho de lombo & 0,72 & $0,34(0,09)$ & & $0,02(0,27)$ & $0,05(0,06)$ \\
Espessura de gordura na garupa & 0,31 & 0,04 & & $0,32(0,02)$ & $0,64(0,22)$ \\
Espessura de gordura na costela & 0,34 & 0,09 & & 0,60 & $0,10(0,08)$ \\
\hline
\end{tabular}

NE: não convergiu para o máximo global.

Para os dois modelos, as estimativas de herdabilidade foram semelhantes. Para peso corporal e área de olho de lombo, os valores de 0,42 ou 0,41 e 0,35 ou 0,34 , respectivamente, são próximos dos encontrados por Figueiredo (2001), que estimou em 0,32 e 0,30, na mesma ordem, ao trabalhar com animais Nelore. Também são semelhantes aos estimados por Moser (1998), de 0,29 e 0,38 , que utilizou dados de 3.583 animais Brangus.

As herdabilidades estimadas para a espessura de gordura na costela e espessura de gordura na garupa são próximas àquelas estimadas por Moser (1998). Observou-se que a variância genética aditiva para espessura de gordura na garupa foi maior do que para espessura de gordura na costela, tornando a característica mensurada na garupa mais vantajosa do ponto de vista prático por apresentar maior componente genético aditivo, e mais indicada para ser utilizada em programas de seleção.

Peso corporal foi favoravelmente correlacionado com a área de olho de lombo $(0,79)$ e, com menor intensidade $(0,20)$, com a espessura de gordura na garupa. A correlação entre peso corporal e a espessura de gordura na costela não convergiu para o máximo global, apesar de várias tentativas com diferentes valores iniciais para os parâmetros. Segundo Bang et al. (1994), a correlação genética entre peso corporal do animal vivo e a espessura de gordura subcutânea, medida com ultrassom, foi 0,78 para 42 touros com pesos entre 260 e $650 \mathrm{~kg}$. Esta mesma correlação, avaliada em carcaças, foi 0,77 , maior que a correlação encontrada na presente pesquisa $(0,20)$, medida na garupa. Bertrand et al. (2001) compilaram dados das correlações genéticas entre área de olho de lombo, espessura de gordura subcutânea e gordura intramuscular, em estudos feitos com animais Bos taurus, publicados desde 1980 e encontraram correlações genéticas médias de -0,04, -0,21 e 0,01 , para a correlação entre AOL e espessura de gordura na costela, espessura de gordura na garupa e gordura marmorizada, respectivamente. Reverter et al. (2000), ao estudarem as raças Angus e Hereford, também encontraram correlações negativas de $-0,07$ e $-0,13$, entre a área de olho de lombo e a espessura de gordura na garupa. Os autores argumentam que o antagonismo genético verificado no estudo serve de alerta, pois os animais com maior área de olho de lombo foram menos precoces em depositar gordura subcultânea, com possível reflexo negativo sobre a precocidade e a fertilidade das fêmeas. Determinada quantidade mínima de gordura corpórea é necessária para a puberdade e concepção (Ball et al., 1995).

A correlação encontrada no presente estudo, entre espessura de gordura na costela e na garupa de, 0,64 , indica que os animais com deposição de gordura subcultânea o fazem tanto na garupa quanto na costela. Alta correlação entre essas duas características também foi obtida por Wilson et al. (2001) que, em experimento com animais Bos taurus, encontraram valor de 0,74 para este parâmetro. Pode-se inferir, com base nos resultados do presente estudo, que programas de melhoramento busquem aumento na espessura de gordura subcutânea podem utilizar somente uma das duas medidas como critério de seleção. Neste sentido, a característica espessura de gordura na garupa apresenta vantagens, pois associa sua grande variabilidade fenotípica à maior variabilidade genética aditiva.

As estimativas das correlações genéticas entre o peso corporal e as características de carcaça permitem conjecturar sobre os efeitos que a 
seleção, tradicionalmente utilizada nos programas de seleção de gado de corte no Brasil, para peso corporal, teria sobre as características de carcaça. Apesar de não haver antagonismo genético entre essas características, e de a seleção para peso corporal estar associada à resposta correlacionada na área de olho de lombo, correspondente a $86,7 \%$ da resposta direta, o impacto da seleção para peso corporal sobre a espessura de gordura é pequeno, 22,8\% do que seria alcançado pela seleção direta.

\section{CONCLUSÕES}

As estimativas de repetibilidade sugerem que as medidas de ultrassonografia no animal podem ser tomadas somente uma vez, reduzindo custos e tempo nos programas de melhoramento. As estimativas de herdabilidade para as características área de olho de lombo e espessura de gordura na garupa sugerem que se pode obter progresso genético com a seleção direta para essas características. A magnitude e direção das correlações genéticas indicam ausência de antagonismo entre a seleção para peso corporal e as características área de olho de lombo e espessura de gordura na carcaça.

\section{REFERÊNCIAS BIBLIOGRÁFICAS}

ARAÚJO, F.R.C. The use of real-time ultrasound to estimate variance components fro growth and carcass traits in Nelore cattle. 2003. 56f. Dissertação (Mestrado) - Department of Animal Science, University of California, Davis.

BAILEY, C.M.; JESEN, J.; ANDERSEN, B.B. Ultrasonic scanning and body measurements for predicting composition and muscle distribution in young Holstein $\times$ Friesian bulls. J. Anim. Sci., v.63, p.1337-1346, 1986.

BALL, A.J.; THOMPSON, J.M.; HINCH, G.N. et al. Feed requirements for maintenance of mature rams and ewes from lines divergently selected for differences in body composition. Proc. N. Z. Soc. Anim. Prod., v.55, p.133-136, 1995.

BANG, K.S.; YOON, J.K.; KIM, Y.K. Ultrasonic backfat measurement in Korean native cattle. Korean J. Anim. Sci., v.36, p.409414, 1994.
BERG, R.T.; BUTTERFIELD, R.M. New concepts of cattle growth. Austrália: Sydney University Press, 1986. 240p.

BERTRAND, J.K.; GREEN, R.D.; HERRING, W.O. et al. Genetic evaluation for beef carcass traits. J. Anim. Sci., v.79, suppl., p.190-200, 2001.

BOLDMAN, K.G.; KRIESE, L.A.; VAN VLECK, L.D. et al. A manual for use for MTDFREML: A set of programs to obtain estimates of variance and covariance. Lincoln: Department of Agriculture, Agricultural Research Service, 1995. 120p.

FIGUEIREDO, L.G.G. Estimativas de parâmetros genéticos de características de carcaças feitas por ultrassonografia em bovinos da raça Nelore. 2001. 67f. Dissertação (Mestrado) - Faculdade de Zootecnia e Engenharia de Alimentos, Universidade de São Paulo, Pirassununga.

LÔBO, R.B.; BEZERRA, L.A.F.; OLIVEIRA, H.N. et al. Avaliação genética de touros e matrizes da raça Nelore: Sumário 2006: Ribeirão Preto: Rochedo Gráfica e Editora, 2006.124p.

LUCHIARI FILHO, A. Pecuária da carne bovina. São Paulo: LinBife, 2000. 134p.

MARCONDES, C.R.; BERGMANN, J.A.G.; ELER, J.P. et al. Análise de alguns critérios de seleção para características de crescimento na raça Nelore. Arq. Bras. Med. Vet. Zootec., v.52, p.83-89, 2000.

MOSER, D.V.; BERTRAND, J.K.; MISZTAL, I. et al. Genetic parameter estimates for carcass and yearling ultrasound measurements in brangus cattle. J. Anim. Sci., v.76, p.2542-2548, 1998.

REVERTER, A.; JOHNSTON, D.J.; GRASER H.U. et al. Genetic analyses of live-animal ultrasound and abattoir carcass traits in Australian Angus and Hereford cattle. J. Anim. Sci., v.78, p.1786-1795, 2000.

REVERTER, A.; JOHNSTON, D.J.; FERGUSON, D.M. et al. Genetic abd phenotypic characterization of animal, carcass, and meat quality from temperate and tropically adapted beef breeds.4. Correlations among animal, carcass, and meat quality traits. Aust. J. Agric. Res., v.54, p.149-158, 2003. 
RITCHIE, H. Available technology tools to produce and deliver final products. In: SYMPOSIUM: USING SCIENCE AND MARKETING IN PRODUCING AND DELIVERING VALUE-ADDED PRODUCTS IN THE NEW ECONOMY, 2001, Bozeman. Proceeding..., Bozeman: Western Section of the American Society of Animal Science, 2001.

SIMONELLI, S.M.; SILVA, M.A.; SILVA, L.O.C. et al. Critérios de seleção para características de crescimento em bovinos da raça Nelore. Arq. Bras. Med. Vet. Zootec., v.56, p.374-384, 2004.
YOKOO, M.J. Estimativas de efeitos genéticos e ambientais para características de carcaça medidas pelo ultra-som em bovinos da raça Nelore. 2005. 89f. Dissertação (Mestrado) Faculdade de Ciências Agrárias e Veterinárias, Universidade Estadual Paulista, Jaboticabal.

WILSON, D.E.; ROUSE, G.H.; HAYS, C.L. Carcass EPDs from Angus heifer real-time ultrasound scan. Ames: Cooperative Extention Service, Iowa State University, 2001. 122p. (Beef Research Report. A.A., Leaflet R1736). 